\title{
FORECAST RESEARCH OF DROPLET SIZE BASED ON GREY THEORY
}

\author{
Ping Liang ${ }^{1,2, *}$, Hongping Zhou ${ }^{1}$, Jiaqiang Zheng ${ }^{1}$ \\ ${ }^{I}$ College of Mechanical and Electrical Engineering, Nanjing Forestry University, Nanjing, \\ China, 210037 \\ ${ }^{2}$ Department of Mechanical Engineering, Huaiyin Institute Technology, Huai'an, \\ China, 223003 \\ * Corresponding author, Address: College of Mechanical and Electrical Engineering, \\ Nanjing Forestry University, 159 longpan Road, Nanjing, 210037, P. R. China, Tel: +86-25- \\ 85442189, Fax: +86-25-85424189, Email: hyitlp@sina.com
}

\begin{abstract}
The droplet size can influence the application of a spray system. We apply grey theory in establishing a model of forecasting droplet size. The example used in this study shows that this method is precise in forecasting the droplet size, and is helpful to choose the other parameters of a spray system within a certain droplet size.
\end{abstract}

Keywords: grey theory, droplet size, forecast

\section{INTRODUCTION}

The effect of spray is determined by the droplet size which is an important index of the function of a sprayer performance. Rapidly and accurately measuring the size and distribution of droplets is required in the study, production, and application of a sprayer. As people deeply realize the influence of droplet size to sprayer performances, the study in droplet size is attached more and more importance. In a spray system, droplet size is affected by the air pressure of the system, the flow, the consistence of the liquid, the angle of spraying, and types of nozzle. Each biological target demands a specific droplet size. The more droplets can be captured on the target, the better the preventing and controlling harmful insect's effect will be. Therefore, accurately measuring droplet size and controlling the 
distribution of droplet size are very important for the understanding and studying the procedure of spraying.

The analysis of droplet size of a spray system is usually based on a great deal of data collected from a large quantity of samples, which is timeconsuming, laborious and is not accurate. Applying grey theory in forecasting droplet size greatly simplifies the routine method and can effectively increase the validity of the results. This method makes best of the internal relevancy of similar samples and gets corresponding analysis data by less samples. At the same time, the other parameters of a spray system corresponding to certain droplet sizes can be forecasted by establishing a grey model.

\section{GREY THEORY}

Grey theory is brought forward and developed by Dr. Julong Deng in the eighties in the twentieth century (Deng, 1997). Its research targets are those objects with incomplete information, uncertain concepts, and mechanisms with uncertain relationship. Its tasks are: (1) Invent a new method of establishing a grey model to overcome the weakness of probability and to find the rule among the confused, limited and discrete data; (2) Use the method to carry out respective analysis, forecast, decision and programming. Grey forecast can be processed with small quantity of data in a short time.

\subsection{Definition and classification of grey forecast}

In grey theory, white refers to the information that is complete awareness, black refers to the information that is scanty and grey refers to the information that is incomplete. The system with incomplete information is a grey system. Grey forecast can find out and master the developing rule of a system, and make out measurable forecast of the target system conditions by processing original data and establishing a grey mathematics model. Usually we build differential equation based on original data so that random disturbance among original data weaken and the contained complete information is strengthened by accumulated generation. The general form of a grey model is GM (n, h), in which $n$ is progression of grey differential equation and $h$ is the number of the variable.

Grey forecasts can be classified into different types of forecasts according to the respective function. For example: sequence forecast, abnormal forecast, topological forecast and system forecast and so on. This work mainly focuses on grey sequence forecast.

\subsection{The building of grey number arrange forecast model}

Grey sequence forecast model GM $(1,1)$, the model used for forecasting time data, is set up in the following steps: 
1 Supposes: there is an initial data as following,

$$
X^{(0)}=\left(x^{(0)}(1), x^{(0)}(2), \cdots, x^{(0)}(n)\right)
$$

$2 X^{(0)}$ is processed by 1-AGO (accumulating generation operator), we can get

$$
\begin{aligned}
& X^{(1)}=\left(x^{(1)}(1), x^{(1)}(2), \cdots, x^{(1)}(n)\right) \\
& \quad X^{(1)}(k)=\sum_{i=1}^{k} x^{(0)}(i), k=1,2, \cdots, n \\
& \text { Therein to, }
\end{aligned}
$$

$3 X^{(1)}$ can establish whitenization function show as

$$
\frac{d x^{(1)}}{d t}+a x^{(1)}=b
$$

Then we calls formula (3) is a one-step and single-variable grey differential equation model $\mathrm{GM}(1,1)$

$4 a, b$ are elements of parameters vector $\hat{a}$, that is

$$
\hat{a}=[a, b]^{T}
$$

Building repeated additive matrix $\mathrm{B}$ and constant vector $Y_{n}$, that is

$$
\begin{aligned}
& B=\left[\begin{array}{cc}
-0.5\left(x^{(1)}(1)+x^{(1)}(2)\right) & 1 \\
-0.5\left(x^{(1)}(2)+x^{(1)}(3)\right) & 1 \\
\ldots & \ldots \\
-0.5\left(x^{(1)}(n-1)+x^{(1)}(n)\right) & 1
\end{array}\right], \\
& Y_{n}=\left[\begin{array}{c}
x^{(0)}(2) \\
x^{(0)}(3) \\
\vdots \\
x^{(0)}(n)
\end{array}\right]
\end{aligned}
$$

5 Make out grey parameter $\hat{a}$ using least square, then

$$
\widehat{a}=\left[\begin{array}{ll}
a & b
\end{array}\right]^{T}=\left[B^{T} B\right]^{-1} B^{T} Y_{n}
$$

6 The value of differential equation in whitenization format is

$$
\bar{X}^{(1)}(k+1)=\left(x^{(0)}(1)-\frac{b}{a}\right) e^{-a k}+\frac{b}{a}
$$

$k=1,2, \cdots, n$

7 Calculate the simulation value of $X^{(1)}$ by the following formula

$$
\widehat{X}^{(1)}=\left(x^{(1)}(1), x^{(1)}(2), \cdots, x^{(1)}(n)\right.
$$

8 Get out the simulation value of $X^{(0)}$ by IAGO (Inverse accumulating generation operator) 


$$
\begin{aligned}
& \hat{X}^{(0)}=\left(\hat{x}^{(0)}(1), \hat{x}^{(0)}(2), \cdots, \hat{x}^{(0)}(n)\right) \\
& \hat{x}^{(0)}=\left(\hat{x}^{(1)}(k)-\hat{x}^{(1)}(k-1)\right.
\end{aligned}
$$

Parameter $a$ is developing coefficient which reflects development trend of $\widehat{X}^{(0)}$ and $\widehat{X}^{(1)}$; Parameter $\mathrm{b}$ is grey action factor which come from background data and reflects variety relation of data.

\subsection{The test of grey sequence forecast model}

The test system forecasts the quality of a grey sequence forecast model. Whether a model can fully satisfy the requirements of practice is not determined until it is been carefully tested. Usually we test a grey forecast model by methods of residual error $(\alpha)$ test, probability of minor error $(\mathrm{p})$ test and degree of grey incidence $(\xi)$ test. All of these methods evaluate the precision of a model by reviewing residual error, a model with high precision demands small average relative error and simulation error, and large relevancy $(\xi)$. The model is considered to be reliable when $\xi>0.60$, the quotient of square ratio $\mathrm{C}$ is minor, and probability of minor error $\mathrm{P}$ is large. For the Given values of $\alpha, \xi, \mathrm{C}$ and $\mathrm{P}$, we can classify forecast precision by good, general, eligibility and bad (shown as Table 1).

Table 1. Class table of testing model precision

\begin{tabular}{lcccc}
\hline Accuracy class & $\begin{array}{c}\text { Residual error } \\
(\alpha)\end{array}$ & $\begin{array}{l}\text { Quotient of } \\
\text { square ratio }(\mathrm{C})\end{array}$ & $\begin{array}{c}\text { Probability } \\
\text { of error }(\mathrm{P})\end{array}$ & $\begin{array}{c}\text { Degree of grey } \\
\text { incidence }(\xi)\end{array}$ \\
\hline Good & $1 \%$ & 0.35 & 0.95 & 0.90 \\
General & $5 \%$ & 0.5 & 0.80 & 0.80 \\
Eligibility & $10 \%$ & 0.65 & 0.70 & 0.70 \\
Bad & $20 \%$ & 0.80 & 0.60 & 0.60 \\
\hline
\end{tabular}

\section{FORECAST OF DROPLET SIZE BASED ON GREY THEORY}

Due to the complexity of forming droplet and variety of spray specification, distribution of droplet size shows a great difference. The distribution of droplet of hydraulic sprayer is determined by detailed analysis. The methods of measuring droplet size include mechanical method, electric method and optical method, among which optical method with a laser droplet analyzer is most accurate. In the process of measuring droplet size, the collection of data is carried out by the laser droplet analyzer of model Winner313. Table 2 is the data collected by the laser droplet analyzer of model Winner313. 
Table 2. Droplet size data by laser droplet analyzer of model Winner313

\begin{tabular}{lllllll}
\hline Water pressure $(\mathrm{MPa})$ & 0.10 & 0.12 & 0.14 & 0.16 & 0.18 & 0.20 \\
\hline Droplet size $(\mu \mathrm{m})$ & 189 & 183 & 176 & 171 & 168 & 162 \\
\hline
\end{tabular}

Table 2 data processing: $X^{(0)}$ carries out one step AGO, then we get the data matrixes B and $Y_{n}$.

$$
B=\left[\begin{array}{cc}
-280.5 & 1 \\
-460 & 1 \\
-633.5 & 1 \\
-803 & 1 \\
-968 & 1
\end{array}\right], Y_{n}=\left[\begin{array}{c}
183 \\
176 \\
171 \\
168 \\
162
\end{array}\right]
$$

Here, $\widehat{a}=\left[\begin{array}{ll}a & b\end{array}\right]^{T}=\left[B^{T} B\right]^{-1} B^{T} Y_{n}=(0.02913,190.3226) \mathrm{T}$,

According to formula (6), we can get

$$
\begin{aligned}
\hat{X}^{(1)}(k+1) & =\left(189-\frac{190.3226}{0.02913}\right) e^{-0.02913 k}+\frac{190.3226}{0.02913} \\
& =-6344.6099 e^{-0.02913 k}+6533.6099
\end{aligned}
$$

Therefore, we can get the simulation value of $X^{(0)}$ by IAGO

$$
\begin{aligned}
\widehat{X}^{(0)} & =\left(\widehat{x}^{(0)}(1), \hat{x}^{(0)}(2), \cdots, \widehat{x}^{(0)}(6)\right) \\
& =(189,182.15,176.92,171.84,166.91,162.12)
\end{aligned}
$$

Meanwhile, we can obtain droplet size under other water pressure value. According to formula (6), when $\mathrm{k}$ is other value, such as $\mathrm{k}=7$, we can also get

$$
\hat{x}^{(0)}(7)=157.46
$$

In the practical measure, when water pressure value is $0.22 \mathrm{MPa}$, droplet size value is $156 \mu \mathrm{m}$.

In order to evaluate the precision of the model forecast, the model value and the actual value error may be examined by using the residual error. The residual error and the relative error may be expressed as:

Residual error $\varepsilon(k)=X_{k}^{(0)}-\hat{X}_{k}^{(0)}, k=1,2, \cdots, n$

Relative error $\Delta k=\left|\varepsilon(k) / X_{k}^{(0)}\right| \times 100 \%, k=1,2, \cdots, n$,

Table 3 shows the application of the forecast model in forecasting the droplet size of a hydraulic spray system. The forecast data and error have been listed. What needs to be explained is that GM $(1,1)$ forecast model is 
Table 3. Droplet size forecasted data and error

\begin{tabular}{ccccc}
\hline$k$ & $X^{(0)}(k)$ & $\hat{X}^{(0)}(k)$ & $\varepsilon(k)$ & $\Delta k$ \\
\hline 1 & 189 & 189 & 0 & 0 \\
2 & 183 & 182.15 & 0.85 & $0.46 \%$ \\
3 & 176 & 176.92 & -0.92 & $-0.52 \%$ \\
4 & 171 & 171.84 & -0.84 & $-0.49 \%$ \\
5 & 168 & 166.91 & 1.09 & $0.65 \%$ \\
6 & 162 & 162.12 & -0.12 & $-0.74 \%$ \\
7 & 156 & 157.46 & -1.46 & $0.94 \%$ \\
\hline
\end{tabular}

usually only used to forecast the monotone increasing function and monotone decreasing function. In the hydraulic spray system, the droplet size distribution (volume distribution) follows the normal distribution (Ma et al. 1999) that is droplet size peak value exists in a spray process. Therefore, in the droplet size forecast, the model may be established according to the process and the monotone interval. And the forecast can be done by the partition forecast and the generalized analysis so that it can satisfy the droplet size forecast demands.

\section{CONCLUSION}

A droplet size forecast model has been established based gray system theory in this paper. The results show that: (1) The predicted values almost matched with the measured values under the water pressure from $0.10 \mathrm{MPa}$ to $0.22 \mathrm{MPa}$ and their relative errors range were between $0.49 \%-0.94 \%$. (2) The method of grey forecast is simple, is high precise in forecast, and is easy to be simulated by a computer. Meanwhile, it can be used for different nozzle types of droplet size gray forecast model systems. Besides the droplet size, it is also helpful for determining other parameters of a spray system.

\section{REFERENCES}

Deng Ju-Long 1997, Grey Control System (second edition). Huazhong University of Science and Technology, (in Chinese).

Liu Si-Feng, Guo Tian-Bang, Dang Yue-Guo 2004, Grey Systems Theory and Applications (third edition). Science Press, (in Chinese).

Ma ChengWei, Yan HeRong, Yuan DongShun, Cui Ying-An 1999, Droplet Size Distribution of Hydraulic Nozzle. Transactions of the Chinese Society of Agricultural Machinery 30: 33-39 (in Chinese). 